Pacific Journal of Mathematics

THE SYMBOLS OF AN ALGEBRA OF PSEUDODIFFERENTIAL 


\title{
THE SYMBOLS OF AN ALGEBRA OF PSEUDODIFFERENTIAL OPERATORS
}

\author{
ELMAR SCHROHE
}

\begin{abstract}
Atiyah and Singer constructed a Fréchet operator algebra $\mathscr{P}_{\infty}$ by closing a set of zero order pseudodifferential operators on a compact manifold $X$ in the topology generated by all the norms of the spaces $L\left(H^{s}\right), s$ real. Each operator in $\mathscr{P}_{\infty}$ has a 'symbol', a function in $C\left(S^{*} X\right)$. Contradicting a statement of Atiyah and Singer and establishing the manifold analogue of a conjecture by $\mathrm{H}$. $\mathrm{O}$. Cordes, it will be shown that each function in $C\left(S^{*} X\right)$ is the symbol of an operator in $\mathscr{P}_{\infty}$.
\end{abstract}

0. Introduction The algebra $\mathscr{P}^{0}$ was defined by Atiyah and Singer [1] as a subalgebra of the zero order pseudodifferential operators on the compact manifold $X$ : they required the operator symbol $p(x, \xi)$ to approach a limit along each ray in $\xi$-direction. $\mathscr{P}_{s}$ is the closure of $\mathscr{P}^{0}$ in $L\left(H^{s}\right)$, the space of bounded operators on the Sobolev space $H^{s}=H^{s}(X)$ for real $s . \mathscr{P}_{\infty}$ is obtained by closing $\mathscr{P}^{0}$ under the Fréchet topology induced by all the $L\left(H^{s}\right)$-norms. These definitions turn out to be equivalent to transferring the $\mathbf{R}^{n}$ concept of $\mathbf{H}$. O. Cordes and E. Hermann [2], Ch. IV, [4], to the case of a compact manifold. $\mathscr{P}_{s} / K\left(H^{s}\right)$ is a commutative $C^{*}$-algebra with unit (here $K\left(H^{s}\right)$ denotes the ideal of compact operators). By the Gelfand-Naimark theorem it is therefore isometrically isomorphic to the space of continuous functions on a compact Hausdorff space, which is known to be homeomorphic to $S^{*} X$, the unit sphere in the cotangent bundle. The symbol of an operator $A$ in $\mathscr{P}_{s}$ is defined as the function corresponding to $A+K\left(H^{s}\right)$. An interesting feature is that neither the symbols nor the symbol space depend on $s$.

Since $\mathscr{P}_{\infty}$ is contained in each $\mathscr{P}_{s}$, the symbols of its operators certainly form a subspace of $C\left(S^{*} X\right)$. In fact, for the somewhat different case $X=\mathbf{R}^{n}$, H. O. Cordes and D. A. Williams [6] proved that at least all those functions are symbols, which are $C^{\infty}$ on the corresponding symbol space. Using a slightly modified version of Egorov's theorem and a technique developed by $\mathrm{H}$. O. Cordes, Theorem 2.1 shows that for each function $a$ in $C\left(S^{*} X\right)$ we can find an operator $A$ in $\mathscr{P}_{\infty}$ with symbol $a$. This contradicts an assertion of Atiyah and Singer [1], p. 513. They stated without proof that the symbol map from $\mathscr{P}_{\infty}$ to $C\left(S^{*} X\right)$ is not surjective. 
On the other hand, in the case $X=\mathbf{R}^{n}$, this result was conjectured by H. O. Cordes (for a slightly different algebra) and proven by Cordes and the author [5].

The symbol of an operator $A$ in $\mathscr{P}_{s}$ governs the Fredholm properties of $A$. Reducing the order to zero does not result in a loss of generality. The case of elliptic (pseudo-) differential operators is easily reduced to studying operators in $\mathscr{P}_{s}$ or $\mathscr{P}_{\infty}$. Moreover, $\mathscr{P}_{\infty}$ shares one of the most important properties of $C^{*}$-algebras: if $A \in \mathscr{P}_{\infty}$ is invertible in $L\left(H^{s}\right)$, then its inverse is also in $\mathscr{P}_{\infty}$. This makes $\mathscr{P}_{\infty}$ a $\Psi$-subalgebra of $L\left(H^{s}\right)$ as defined by B. Gramsch [7].

Acknowledgment. This subject was suggested to me by Prof. H. O. Cordes when I was at the University of California, Berkeley, as part of my dissertation project. I would like to thank him and Dr. Lars Andersson for many helpful discussions. I am also grateful to the referee. His suggestions led to a simplification in the final part of the proof.

1. Definitions and preliminary results. Throughout this paper $X$ denotes a compact $n$-dimensional manifold. We cover $X$ by a finite set $V_{j}$, $j=1, \ldots, N$ of coordinate neighborhoods which are mapped diffeomorphically onto open, relatively compact sets $U_{j}$ in $\mathbf{R}^{n}$ by charts $\chi_{j}$. We further choose a fixed partition of unity $\left\{\phi_{j}\right\}$ subordinate to $\left\{V_{j}\right\}$ and functions $\psi_{j} \in \mathscr{D}\left(V_{j}\right)$ with $\phi_{j} \cdot \psi_{j}=\phi_{j}$. We choose a Riemannian metric $g=\left(g_{j k}\right)_{j, k=1, \ldots, n}$ on $X$ and define $\left(g^{j k}\right)=\left(g_{j k}\right)^{-1}$, where the matrices correspond to the choice of the coordinates. Unless stated otherwise, i.e. in $1.1,1.2$ or 1.3 , all notions connected with pseudodifferential operators will be used as in M. Taylor's book [10].

1.1. Definition. (a) Let $H^{s}\left(\mathbf{R}^{n}\right)$ be the usual Sobolev space of order $s$ on $\mathbf{R}^{n}$ and $H^{s}=H^{s}(X)$ the Sobolev space on $X$ defined via the fixed partition of unity and the coordinate maps. $H^{s}$ is a Hilbert space.

(b) $L\left(H^{s}\right)$ is the set of all bounded linear operators on $H^{s}$. We will also write $L_{s} . K\left(H^{s}\right)$ or $K_{s}$ denotes the ideal of compact operators on $H^{s}$.

(c) $C B^{\infty}\left(\mathbf{R}^{n} \times \mathbf{R}^{n}\right)$ is the set of all $C^{\infty}$ functions on $\mathbf{R}^{n} \times \mathbf{R}^{n}$ with all derivatives bounded.

(d) For real $m$ let $S^{m}=S^{m}\left(\mathbf{R}^{n} \times \mathbf{R}^{n}\right)$ be the set of all $C^{\infty}$ functions $a$ on $\mathbf{R}^{n} \times \mathbf{R}^{n}$ with

$$
\left\langle x^{\alpha}\right\rangle\left|D_{x}^{\beta} D_{\xi}^{\gamma} a(x, \xi)\right| \leq C_{\alpha \beta \gamma}\langle\xi\rangle^{m-|\gamma|}
$$

for all $x, \xi$ in $\mathbf{R}^{n}$ and all multiindices $\alpha, \beta, \gamma$ with a suitable constant $C_{\alpha \beta \gamma}$. Similarly, $S^{m}\left(U \times R^{n}\right)$ is defined for an open set $U$ in $\mathbf{R}^{n}$ : we 
require $a$ to have its $x$-support in $U$. Note that we used the notation $\langle x\rangle=\left(1+|x|^{2}\right)^{1 / 2}$.

1.2. Definition. (a) Let $a(x, \xi) \in C B^{\infty}\left(\mathbf{R}^{n} \times \mathbf{R}^{n}\right)$. We define the operator $\operatorname{Op}(a)$ on $\mathscr{D}\left(\mathbf{R}^{n}\right)$ by

$$
\operatorname{Op}(a) f(x)=(2 \pi)^{-n / 2} \int e^{i x \xi} a(x, \xi) \hat{f}(\xi) d \xi
$$

where $\hat{f}$ denotes the Fourier transform of $f$. By a slight extension of a theorem due to Calderon and Vaillancourt (cf. [3], Thm. $\mathrm{B}_{\rho}$, p. 117) this extends to a bounded linear operator on each $H^{s}\left(\mathbf{R}^{n}\right)$. We even have the estimate for its norm

$$
\|\mathrm{Op}(a)\|_{s, s} \leq c_{s} \max \left\{\left|D_{x}^{\alpha} D_{\xi}^{\beta} a(x, \xi)\right|:|\alpha|,|\beta| \leq n_{s}\right\},
$$

where $c_{s}, n_{s}$ depend on $s$ but not on $a$.

(b) Similarly we may substitute a function $a \in S^{m}$ into (1.1). Op(a) will then define a bounded operator from $H^{s+m}\left(\mathbf{R}^{n}\right)$ to $H^{s}\left(\mathbf{R}^{n}\right)$ for any real $s$.

(c) Whenever we can represent an operator $A$ in the form $A=\operatorname{Op}(a)$ with a function $a$ in $C B^{\infty}\left(\mathbf{R}^{n} \times \mathbf{R}^{n}\right)$ or $a$ in $S^{m}\left(\mathbf{R}^{n} \times \mathbf{R}^{n}\right)$, we will call $a$ the operator symbol of $A$ in order to avoid confusion with the "symbol" of $A$ which will be defined in 1.4.

(d) This concept is transferred to the manifold case in the usual way. In particular, we will write $P \sim \mathrm{Op}(p)$ if

(i) $p=\left\{p_{j}: p_{j} \in S^{m}\left(U_{j} \times \mathbf{R}^{n}\right)\right\}$ and

(ii) $\Psi_{j} P \Phi_{j} \sim M_{\psi_{j} \circ \chi_{j}^{-1}} \mathrm{Op}\left(p_{j}\right) M_{\phi_{j} \circ \chi_{j}^{-1}}$.

where $\Phi_{j}: C^{\infty}\left(\mathbf{R}^{n}\right) \rightarrow \mathscr{D}\left(V_{j}\right)$ is defined by $\left(\Phi_{j} f\right)(x)=\phi_{j}(x) f\left(\chi_{j}(x)\right)$ and $\Psi_{j}: \quad C^{\infty}(X) \rightarrow \mathscr{D}\left(U_{j}\right)$ by $\left(\Psi_{j} f\right)(y)=\psi_{j}\left(\chi_{j}^{-1}(y)\right) f\left(\chi_{j}^{-1}(y)\right)$. the " $\sim$ " means that the difference is a regularizing operator on $\mathbf{R}^{n}$.

1.3. Proposition. (a) On $T^{*} X$, the cotangent bundle of $X$, a norm is defined by

$$
|\xi|=\left(\sum g^{j k}(x) \xi_{j} \xi_{k}\right)^{1 / 2}
$$

where $\xi \in T_{x}^{*} X$ has the representation $\xi=\sum \xi_{j} d \chi^{j}$, and $\chi$ stands for one of the coordinate maps $\chi_{j}$.

(b) The Laplace-Beltrami operator on $X$ is given in local coordinates by $\Delta=(1 / v) \sum_{j, k} \partial / \partial x_{j} v g^{j k}(x) \partial / \partial x_{k}$, with $v=\left(\operatorname{det} g_{j k}(x)\right)^{1 / 2}$.

(c) The principal symbol of $-\Delta$ is $p(x, \xi)=\sum g^{j k}(x) \xi_{j} \xi_{k}$. Here we use the notion "principal symbol" in the sense that $(-\Delta)-O p(p)$ is an 
operator of order $\leq 1$. It follows from the transformation law for pseudodifferential operators on a manifold that each principal symbol may be considered as a function on the cotangent bundle $T^{*} X$.

(d) The operators $\Lambda, M$ on $X$ are given by

$$
\begin{aligned}
& \Lambda=(Q-\Delta)^{1 / 2} \\
& M=(Q-\Delta)^{1 / 4} .
\end{aligned}
$$

$Q$ denotes the projection onto the constants.

(e) By [9, §4, Thm. 2 and Thm. 3], we know that $\Lambda$ and $M$ are invertible elliptic pseudodifferential operators of order 1 and $\frac{1}{2}$, resp. Their principal symbols are

$$
\begin{aligned}
& \lambda_{0}(x, \xi)=\left(\sum g^{j k}(x) \xi_{j} \xi_{k}\right)^{1 / 2}=|\xi| \text { and } \\
& \mu_{0}(x, \xi)=\left(\sum g^{j k}(x) \xi_{j} \xi_{k}\right)^{1 / 4}=|\xi|^{1 / 2} .
\end{aligned}
$$

(f) Although $\lambda_{0}$ and $\mu_{0}$ are not smooth for $\xi=0$, we can still use them as principal symbols: if we restrict $\lambda_{0}, \mu_{0}$ to $\{|\xi| \geq c\}$ and then extend them smoothly to $\{|\xi|<c\}$ by $\tilde{\lambda}$, $\tilde{\mu}$, then $\operatorname{Op}\left(\lambda_{0}\right)-\operatorname{Op}(\tilde{\lambda})$ and $\operatorname{Op}\left(\mu_{0}\right)-$ $\mathrm{Op}(\tilde{\mu})$ are regularizing.

1.4. Definition. (a) In [1, p. 509], Atiyah and Singer define the class $\mathscr{P}^{0}$. It denotes the set of operators $P$ in $L\left(H^{0}\right)$ with $P \sim \mathrm{Op}(p)$ for an operator symbol $p=\left\{p_{j}: p_{j} \in S^{0}\left(U_{j} \times \mathbf{R}^{n}\right)\right\}$, where each $p_{j}$ has a limit

$$
\sigma\left(p_{j}\right)(x, \xi)=\lim _{t \rightarrow \infty} p_{j}(x, t \xi), \quad \xi \neq 0 .
$$

(b) By patching coordinate neighborhoods together, $\sigma(p)$ is well-defined as a function on $T^{*} X-\{0\}$. It is called the symbol of $P$. We will also write $\sigma(P)$. This is justified by the following lemma which states the basic properties of these symbols.

1.5. LEMMA. The notation is as in 1.4 .

(a) $\sigma\left(p_{j}\right) \in C^{\infty}\left(U_{j} \times\left(\mathbf{R}^{n}-\{0\}\right)\right)$.

(b) In fact, the assumptions in 1.4(a) imply that all derivatives $D^{\alpha} \sigma\left(p_{j}\right)(x, \xi)$ exist on $U_{j} \times\left(\mathbf{R}^{n}-\{0\}\right)$ and that $D^{\alpha} \sigma\left(p_{j}\right)(x, \xi)=$ $\lim _{t \rightarrow \infty} D^{\alpha} p_{j}(x, t \xi), \xi \neq 0$.

(c) Convergence in (b) is uniform: given $\varepsilon>0$ and a fixed pair $\left(x_{0}, \xi_{0}\right)$ in $U_{j} \times\left(\mathbf{R}^{n}-\{0\}\right)$, there is $a t_{0}>0$ and a neighborhood $U$ of $\left(x_{0}, \xi_{0}\right)$ such that

$$
\left|D^{\alpha} p_{j}(x, t \xi)-D^{\alpha} \sigma\left(p_{j}\right)(x, \xi)\right|<\varepsilon
$$

for all $(x, \xi)$ in $U$ and $t \geq t_{0}$. 
(d) $\sigma\left(p_{j}\right)$ is homogeneous of degree 0 in $\xi$.

(e) By patching together the coordinate neighborhoods with the partition of unity, $\sigma(p)$ is well-defined on $T^{*} X-\{0\}$.

(f) For $A, B$ in $\mathscr{P}^{0}, t \in \mathbf{C}$, we have

$$
\begin{aligned}
\sigma(A+B) & =\sigma(A)+\sigma(B) \\
\sigma(A B) & =\sigma(A) \sigma(B) \\
\sigma(t A) & =t \sigma(A) \\
\sigma\left(A^{*}\right) & =\overline{\sigma(A)} .
\end{aligned}
$$

In particular, all operators on the left hand side of these equations are in $\mathscr{P}^{0}$, and $\mathscr{P}^{0}$ is a*-closed subalgebra of $L_{0}$.

(g) If two operators in $\mathscr{P}^{0}$ have the same symbol, then their difference is compact.

Proof. (a) follows from (b).

(b) is proved by induction. To make things easier, we may assume $n=1$ and show only

(i) $\partial_{x} p_{j}(x, t \xi)$ approaches a limit $q(x, \xi)$ as $t \rightarrow \infty, \xi \neq 0$, and so do all other derivatives with respect to $x$ or $\xi$.

(ii) $q=\partial_{x} \sigma\left(p_{j}\right)$.

First suppose (i) did not hold in $\left(x_{0}, \xi_{0}\right)$, i.e. $\partial_{x} p_{j}\left(x_{0}, t \xi\right)$ did not approach a limit as $t \rightarrow \infty$. By the usual simplifications we may assume that $p_{j}$ is real-valued and that we have a sequence $t_{1}, t_{2}, \ldots, \rightarrow \infty$ such that $\partial_{x} p_{j}\left(x_{0}, t \xi_{0}\right)>1$ for $k$ even and $<-1$ for $k$ odd. Since $\partial_{x x} p_{j}$ is bounded on $U_{j} \times \mathbf{R}^{n}$, there is a $c>0$, such that the above inequalities also hold for all $x$ with $\left|x-x_{0}\right|<c$. We conclude that

$$
p_{j}\left(x_{0}+c, t_{k} \xi_{0}\right)-p_{j}\left(x_{0}, t_{k} \xi_{0}\right)=c \int \partial_{x} p_{j}\left(x_{0}+u c, t_{k} \xi_{0}\right) d u,
$$

which is $>c$ for $k$ even and $<-c$ for $k$ odd. Hence one of the sequences on the left hand side cannot converge as $k \rightarrow \infty$, contradicting our assumption. A similar argument now holds for all other derivatives.

To prove (ii), we first note that

$$
\begin{aligned}
\frac{\left(\sigma\left(p_{j}\right)(x+h, \xi)-\sigma\left(p_{j}\right)(x, \xi)\right)}{h}-q(x, \xi) \\
=h \lim _{h \rightarrow \infty} \int_{0}^{1} \int_{0}^{1} \partial_{x x} p_{j}(x+u v h, t \xi) d u d v \\
=h \int_{0}^{1} \int_{0}^{1} \lim \partial_{x x} p_{j}(x+u v h, t \xi) d u d v
\end{aligned}
$$


by Lebesgue's theorem on dominated convergence, since $\partial_{x x} p_{j}$ is bounded and approaches a limit as $t \rightarrow \infty$. Thus we can take the limit $h \rightarrow 0$ on the left hand side, and it equals zero.

(c) We pick an arbitrary $\left(x_{0}, \xi_{0}\right), \xi_{0} \neq 0$, let $\varepsilon>0$ be given and write for the moment $\sigma^{*}$ instead of $D^{\alpha} \sigma\left(p_{j}\right)$ and $p^{*}$ instead of $D^{\alpha} p_{j}$. By (b), $\sigma^{*}$ is continuous. This furnishes a neighborhood $U_{1}$ of $\left(x_{0}, \xi_{0}\right)$ such that $\left|\sigma^{*}(x, \xi)-\sigma^{*}\left(x_{0}, \xi_{0}\right)\right|<\varepsilon$ for $(x, \xi)$ in $U_{1}$. On the other hand,

$$
\begin{aligned}
& \left|p^{*}(x, t \xi)-p^{*}\left(x_{0}, t \xi_{0}\right)\right| \\
& \quad \leq \int_{0}^{1}\left\|\operatorname{grad}\left(p^{*}\left(x_{0}+u\left(x-x_{0}\right), t \xi_{0}+u\left(\xi-\xi_{0}\right)\right)\right)\right\| \\
& \quad \times\left|\left(x-x_{0}, t\left(\xi-\xi_{0}\right)\right)\right| d u \\
& \quad \leq \int_{0}^{1}\left\langle t \xi_{0}+u t\left(\xi-\xi_{0}\right)\right\rangle^{-1}\left|\left(x-x_{0}, t\left(\xi-\xi_{0}\right)\right)\right| d u \\
& \quad=O\left(t^{-1}\left|x-x_{0}\right|+\left|\xi-\xi_{0}\right|\right) \quad \text { for all } t>1, \quad x \in U_{j}, \xi \in \mathbf{R}^{n} .
\end{aligned}
$$

In the second inequality we used the fact that $p^{*}$ is in $S^{0}$ (even in $S^{-|\alpha|}$ ) and therefore satisfies an inequality of the form stated in 1.1(d). Hence we can find a neighborhood $U_{2}$ of $\left(x_{0}, \xi_{0}\right)$ such that $\left|p^{*}(x, t \xi)-p^{*}\left(x_{0}, t \xi_{0}\right)\right|$ $<\varepsilon$ for all $t \geq 1,(x, \xi) \in U_{2}$. Finally there is a $t_{0} \geq 1$ with $\mid p^{*}\left(x_{0}, t \xi_{0}\right)$ $-\sigma^{*}\left(x_{0}, \xi_{0}\right) \mid<\varepsilon$ for $t \geq t_{0}$. So, if we choose $(x, \xi)$ in $U=U_{1} \cap U_{2}$ and $t \geq t_{0}$ we obtain

$$
\begin{aligned}
& \left|p^{*}(x, t \xi)-\sigma^{*}(x, \xi)\right| \\
& \quad \leq\left|p^{*}(x, t \xi)-p^{*}\left(x_{0}, t \xi_{0}\right)\right|+\left|p^{*}\left(x_{0}, t \xi_{0}\right)-\sigma^{*}\left(x_{0}, \xi_{0}\right)\right| \\
& \quad+\left|\sigma^{*}\left(x_{0}, \xi_{0}\right)-\sigma^{*}(x, \xi)\right|<3 \varepsilon .
\end{aligned}
$$

This proves (c).

(d) is obvious.

(e) follows from the transformation law for pseudodifferential operators under diffeomorphisms on $\mathbf{R}^{n}$, cf. [10, §II.5].

(f) is a consequence of the calculus of pseudodifferential operators, $\mathrm{cf}$. [10, §II.4].

(g) By (f) we may assume that $P \in \mathscr{P}^{0}$ has symbol zero. We have to show that $P$ is compact. Using the fact that $P \sim \mathrm{Op}(p)$, which is made up from a finite number of operators $\operatorname{Op}\left(p_{j}\right)$ on $\mathbf{R}^{n}$, we may confine ourselves to the case $P=\operatorname{Op}\left(p_{j}\right), p_{j} \in S^{0}\left(U_{j} \times \mathbf{R}^{n}\right)$ with $\sigma\left(p_{j}\right)=0$. Now choose a sequence of functions $\left(h_{k}\right)_{k}$ in $\mathscr{D}\left(\mathbf{R}^{n}\right)$ with $h_{k}(\xi)=1$ for 
$|\xi| \leq k$, and $\left|h_{k}^{(\alpha)}(\xi)\right| \leq 1$ for all $|\alpha| \leq n_{0}$, where $n_{0}$ is the constant in 1.2(a). We write

$$
\mathrm{Op}\left(p_{j}\right)=\mathrm{Op}\left(h_{k} p_{j}\right)+\mathrm{Op}\left(\left(1-h_{k}\right) p_{J}\right)
$$

The first operator on the right hand side is regularizing, since it has compact support in both, $x$ and $\xi$. In particular, it is compact, since $X$ is compact. As for the second operator, we first note that $\left(1-h_{k}\right) p_{j}$ is in $C B^{\infty}\left(\mathbf{R}^{n} \times \mathbf{R}^{n}\right)$ with

$$
\begin{aligned}
\sup \left\{\left|D^{\alpha}\left(\left(1-h_{k}\right)(\xi) p_{j}(x, \xi)\right)\right|:|\alpha| \leq n_{0}, x \in U_{j}, \xi \in \mathbf{R}^{n}\right\} \\
\quad=\sup \left\{\left|D^{\alpha} p_{j}(x, \xi)\right|:|\alpha| \leq n_{0}, x \in U_{j},|\xi| \geq k\right\} .
\end{aligned}
$$

This tends to zero as $k \rightarrow \infty$ : The closure $C$ of the set $\left\{(x, \xi) \in U_{j} \times \mathbf{R}^{n}\right.$ : $p(x, \xi) \neq 0,1 \leq|\xi| \leq 2\}$ is compact. Thus it can be covered by finitely many of the neighborhoods constructed in (c). This gives us a $t_{0}>0$ such that $\left|D^{\alpha} p_{j}(x, t \xi)\right|<\varepsilon$ for all $(x, \xi)$ in the set $C, t \geq t_{0},|\alpha| \leq n_{0}$, or $\left|D^{\alpha} p_{j}(x, \xi)\right|<\varepsilon$ for all $x \in U_{j},|\xi| \geq t_{0}$. Using the Calderon-Vaillancourt theorem, we see that the second operator on the right hand side tends to zero. Therefore, $P$ is compact.

1.6. Definition (cf. [1], p. 512). $\mathscr{P}_{s}$ denotes the closure of $\mathscr{P}^{0}$ in $L_{s}$, $\mathscr{P}_{\infty}$ the closure of $\mathscr{P}^{0}$ in the topology generated by the set of norms $\left\{\|\cdot\|_{s, s}: s \in \mathbf{R}\right\}$ of operators on all spaces $H^{s}$. It follows from Calderon's Interpolation Theorem (cf. [10], p. 22) that this is indeed a Fréchet topology.

1.7. REMARK. Let $M$ be the operator defined in 1.3(d).

(a) For $P \in \mathscr{P}^{0}, k$ an integer, we have $M^{k} P M^{-k}-P \in K_{s}$ for all $s$.

(b) $M^{k} \mathscr{P}_{s} M^{-k}=\mathscr{P}_{s-k / 2}, k \in \mathbf{Z}, s \in \mathbf{R}$ $M^{k} K_{s} M^{-k}=K_{s-k / 2} k \in \mathbf{Z}, s \in \mathbf{R}$.

(c) Let $P \in \mathscr{P}_{0}$. Then $P \in \mathscr{P}_{\infty}$ if and only if $M^{k} P M^{-k}-P \in K_{0}$ for all $k \in \mathbf{Z}$.

(d) Let $P \in \mathscr{P}_{0}$. Then

$P \in \mathscr{P}_{\infty}$ if and only if $\mathrm{ad}^{k} M(P) M^{-k}, M^{-k} \mathrm{ad}^{k} M(P) \in K_{0}$ for $k \in \mathbf{N}$. Here $\operatorname{ad}^{k} M(P)$ denotes the $k$-fold commutator of $P$ with $M:[M, \ldots,[M, P] \ldots]$.

(e) $\mathscr{P}_{s}$ contains $K_{s}$ for all $s$.

Proof. (a) follows from the calculus of pseudodifferential operators: the difference is an operator of order $\leq-1$ and hence compact. 
(b) For each real $k, M^{k}$ is an isomorphism of $H^{s}$ onto $H^{s-k / 2}$. This leads to an estimate of the norms of the operators involved on $H^{s}$ and $H^{s-k / 2}$. Since $\mathscr{P}_{s}$ is defined as the closure of $\mathscr{P}^{0}$ and $K_{s}$ is the closure of the finite dimensional operators, this yields the desired result.

(c) The proof is a slight modification of the proof given in [2], Lemma IV 5.3 for the $\mathbf{R}^{n}$-case.

(d) follows from (c) by induction.

(e) cf. [1], p. 512.

1.8. REMARK. Similar as in [5], we might introduce the algebra $\mathscr{A}^{0}$ generated by

(i) The multiplication operators $M_{\phi}, \phi \in C^{\infty}(X)$ and

(ii) the $n+1$ operators $\sum \Psi_{j} \mathrm{Op}\left(\xi_{k} /\langle\xi\rangle\right) \Phi_{j}, \sum \Psi_{j} \mathrm{Op}\left(1 /\langle\xi\rangle^{2}\right) \Phi_{j}, k=$ $1, \ldots, n$. The algebras $\mathscr{A}_{s}$ are the closure of $\mathscr{A}^{0}$ in $L_{s}, \mathscr{A}_{\infty}$ is the closure in the Fréchet topology. Using 1.5 and the Stone-Weierstrass theorem it can be shown that $\mathscr{A}_{s}=\mathscr{P}_{s}, \mathscr{A}_{\infty}=\mathscr{P}_{\infty}$.

1.9. THEOREM. $\mathscr{P}_{s} / K_{s}$ is a commutative $C^{*}$-algebra with unit for each real s. $\mathscr{P}_{s} / K_{s} \simeq C\left(S^{*} X\right)$, the space of continuous functions on the unit sphere in the cotangent bundle.

Proof. [1], p. 512 or $[\mathbf{8}]$.

1.10. Remark. For $A$ in $\mathscr{P}_{s}$, the function $a$ associated with $A+K_{s}$ via the Gelfand isomorphism is called the symbol of $A$. For $A$ in $\mathscr{P}^{0}$ it turns out that $a$ is just the restriction of $\sigma(A)$ as defined in 1.4 to $S^{*} X$. Since we know that $\sigma(A)$ is homogeneous of degree 0 , we may ignore this difference (cf. [1], [8])

1.11. Definition. We consider the strictly hyperbolic time-independent pseudodifferential equation

$$
\begin{gathered}
\partial_{t} u=i M u \\
u(s, \cdot)=g \in H^{k}(X), \quad k \text { real. }
\end{gathered}
$$

(a) By $S(t, s)$ we denote the operator on $H^{k}(X)$ taking $g=u(s, \cdot)$ to $u(t, \cdot)$ and by $S(t)$ the operator $S(t, 0)$.

(b) $H$ is the Hamiltonian vector field on $T^{*} X$ induced by the principal symbol $\mu_{0}$ of $M$, homogeneously extended to zero, cf. 1.3(e), (f):

$$
H=\left(\frac{\partial \mu_{0}}{\partial \xi_{j}} \frac{\partial}{\partial x_{j}}-\frac{\partial \mu_{0}}{\partial x_{j}} \frac{\partial}{\partial \xi_{j}}\right) \text {. }
$$


(c) $F_{t}$ is the flow generated by $H$. For $\left(x_{0}, \xi_{0}\right)$ in $T^{*} X$ we shall also write $F_{t}\left(x_{0}, \xi_{0}\right)=\left(x_{t}\left(x_{0}, \xi_{0}\right), \xi_{t}\left(x_{0}, \xi_{0}\right)\right)=\left(x_{t}, \xi_{t}\right)$.

1.12. LeMMA. (a) The solution to equations (1.2) exists and is uniquely determined in $H^{k}(X)$. The operator $S(t)$ is bounded on each $H^{k}(X)$, $t, k \in \mathbf{R}$.

(b) $S(t+s, s)=S(t, 0)=S(t)$ for all $s, t$. In particular, $S(-t)=$ $S(-t, 0)=S(0, t)$. $S(t)$ is often written as $e^{i M t}$.

(c) $M$ and $S(t)$ commute.

(d) The flow $F_{t}$ exists for all $t$, and for each $T>0$ there are constants $c$, C such that

$$
0<c \leq\left\langle x_{t}\right\rangle /\left\langle x_{0}\right\rangle,\left\langle\xi_{t}\right\rangle /\left\langle\xi_{0}\right\rangle \leq C \text { for }-T \leq t \leq T .
$$

Proof. (a) Using the notation of Taylor ([10]), $i M$ is symmetric hyperbolic in $\operatorname{OPS}_{1,0}^{1}$ and $\left(i M+i M^{*}\right) \in \mathrm{OPS}_{1,0}^{0}$. Applying Theorem [10, IV2.3] we get existence and uniqueness of the solution. Boundedness is stated in equation IV(2.4).

(b) $S(t, s) g=u(t, \cdot)$, where $u$ satisfies equation (1.2), $s, t$ are fixed. So $S(t, 0) g=u(t, \cdot)$, where $u(0, \cdot)=g$. Introduce $v(y, x)=u(y-s, x)$. Then $\partial_{t} v=\partial_{t} u(y-s, x)=i M u(y-s, x)=i M v$, and $v(s, x)=$ $u(s-s, x)=g(x)$. Thus $S(t+s, s) g=v(t+s, \cdot)=u(t, \cdot)=S(t, 0) g$.

(c) Let $g \in C^{\infty}(X)$, $u$ the solution of equation (1.2) with $s=0$. Then $\partial_{t} u=i M u, u(0, \cdot)=g$. Hence $M S(t) g=M u(t, \cdot)$. On the other hand, $\partial_{t}(M u)=M \partial_{t} u$, since $u \in C^{\infty}(X), \partial_{t}$ and $\Delta$ commute, and $M$ is a holomorphic function of $\Delta$. This implies that $\partial_{t}(M u)=M \partial_{t} u=i M(M u)$, $M u(0, \cdot)=M g$. We see that $S(t) M g=M u(t, \cdot)=M S(t) g$. The density of $C^{\infty}(X)$ in each $H^{k}$ then furnishes the desired result.

(d) For each coordinate neighborhood $V_{j},\left.T^{*} X\right|_{V}$ is mapped diffeomorphically to $U_{j} \times \mathbf{R}^{n}$. The flow $F_{t}$ is the solution of the ODE

$$
\begin{gathered}
\dot{x}=\partial_{\xi} \mu_{0}, x(0)=x_{0}, \\
\dot{\xi}=-\partial_{x} \mu_{0}, \xi(0)=\xi_{0} .
\end{gathered}
$$

It is known that the solution to this ODE exists for small $|t|$ and can be continued to the boundary of $\mathbf{R}^{n} \times U_{j} \times \mathbf{R}^{n}$. Since $\left|\partial_{x} \mu_{0}(x, \xi)\right| \leq$ const. $\langle\xi\rangle^{1 / 2}$ for all $(x, \xi)$ in $U_{j} \times \mathbf{R}^{n}, \xi_{t}$ cannot tend to infinity for finite $t$. Now $F_{t+t_{0}}\left(x_{0}, \xi_{0}\right)=F_{t}\left(x_{t_{0}}, \xi_{t_{0}}\right)$ and $\mu_{0}$ is invariantly defined on $T^{*} X$ (under changes of coordinates). So we can switch to another coordinate neighborhood in case $x_{t}$ approaches the boundary of $U_{j}$. We obtain a 
solution for all $t \in \mathbf{R}$. As for the estimate, we first derive on $U_{j} \times \mathbf{R}^{n}$

$$
\begin{aligned}
& \left\langle x_{t}\right\rangle^{\cdot}=x_{t} \dot{x}_{t} /\left\langle x_{t}\right\rangle=\partial_{\xi} \mu_{0}\left(x_{t}, \xi_{t}\right) x_{t} /\left\langle x_{t}\right\rangle=O\left(\left\langle x_{t}\right\rangle\right) \quad \text { and } \\
& \left\langle\xi_{t}\right\rangle^{\cdot}=\xi_{t} \dot{\xi}_{t} /\left\langle\xi_{t}\right\rangle=-\partial_{x} \mu_{0}\left(x_{t}, \xi_{t}\right) \xi_{t} /\left\langle\xi_{t}\right\rangle=O\left(\left\langle\xi_{t}\right\rangle\right) .
\end{aligned}
$$

We may integrate this to obtain

$$
\begin{aligned}
& \log \left(\left\langle x_{t}\right\rangle /\left\langle x_{0}\right\rangle\right)=O(1), \\
& \log \left(\left\langle\xi_{t}\right\rangle /\left\langle\xi_{0}\right\rangle\right)=O(1) .
\end{aligned}
$$

with constants depending on the bounds for the derivatives of $\mu_{0}$ and $T$. Hence we can keep the constants when switching from one coordinate patch to another and get the global estimate.

1.13. Lemma. Let $f \in C^{\infty}(X)$ be fixed. Then the function $H: \mathbf{R} \rightarrow$ $C^{\infty}(X)$, defined by $H(t)=S(t) f$ is differentiable and $H^{\prime}(t)=\partial_{t} S(t) f=$ $i M S(t) f$, where convergence holds in $C^{\infty}$-topology.

Proof. This follows from the fact that $S(t) f=u(t, \cdot)$, where $u$ satisfies equation (1.2) with $s=0, g=f$, and that a solution is in $C^{m}\left(\mathbf{R}, H^{k-m}\right)(X)$ for each choice of $k, m$, cf. [10, Ch. IV].

1.14. TheOREM. Let $P \sim \mathrm{Op}(p)$ be a pesudodifferential operator of order $m$ on $X$. Then the operator $P(t)=S(-t) P S(t)$ is a pseudodifferential operator on $X$ of order $m$ with principal symbol $p_{t}\left(x_{0}, \xi_{0}\right)=p\left(F_{t}\left(x_{0}, \xi_{0}\right)\right)$

Proof. The proof is the same as the one given in [10, §VIII.1]: M. Taylor's assumptions on the operator symbol may be reduced to those the operator symbol of $M$ satisfies.

1.15. Lemma. Let $t \in \mathbf{R},\left(x_{0}, \xi_{0}\right) \in T^{*} X-\{0\}$ be fixed. If we have the solution

$$
F_{t}\left(x_{0}, \xi_{0}\right)=\left(x_{t}\left(x_{0}, \xi_{0}\right), \xi_{t}\left(x_{0}, \xi_{0}\right)\right)
$$

for small $|t|$, then

$$
F_{t}\left(x_{0}, \rho \xi_{0}\right)=\left(x_{0}+u\left(\rho^{-1 / 2} t\right), \rho\left(\xi_{0}+v\left(\rho^{-1 / 2} t\right)\right)\right),
$$

where $u(t)=x_{t}-x_{0}, v(t)=\xi_{t}-\xi_{0}$, and $\rho$ is sufficiently large.

Proof. For any pair of initial values $\left(x_{0}, \xi_{0}\right), x_{t}$ and $\xi_{t}$ are uniquely determined by solving the ODE stated in the proof of 1.12(d). Define $u(t)$ and $v(t)$ for small values of $|t|$ as in the statement (we only restrict $t$, 
because we do not want the solution to leave the coordinate neighborhood). For given $\rho>0$, define

$$
\begin{aligned}
& \tilde{x}(t)=x_{0}+u\left(\rho^{-1 / 2} t\right), \\
& \tilde{\xi}(t)=\xi_{0}+v\left(\rho^{-1 / 2} t\right),
\end{aligned}
$$

which is possible for small $\rho^{-1 / 2}|t|$. Then $(\tilde{x}(t), \tilde{\xi}(t))$ solves the corresponding ODE with the initial value $\left(x_{0}, \rho \xi_{0}\right)$. By uniqueness, $\tilde{x}(t)=$ $x_{t}\left(x_{0}, \rho \xi_{0}\right), \tilde{\xi}(t)=\xi_{t}\left(x_{0}, \rho \xi_{0}\right)$.

\section{Result and Proof.}

2.1. ThEOREM. Given a in $C\left(S^{*} X\right)$, there is an operator $A$ in $\mathscr{P}_{\infty}$ with $\sigma(A)=a$.

The proof is broken down into a series of lemmata.

\subsection{LEMMA. For $A$ in $\mathscr{P}^{0}$}

(a) $A(t)=S(-t) A S(t) \in \mathscr{P}^{0}, \sigma(A(t))=\sigma(A)$.

(b) $t \mapsto A(t)$ is differentiable from $\mathbf{R}$ into $\mathscr{P}^{0}$ equipped with the $L_{0}$-norm. $\partial_{t} A(t)=i[A(t), M], \sigma\left(\partial_{t} A(t)\right)=-i\left\{\mu_{0}, a\right\}=-i H a$.

Proof. Let $A \sim \mathrm{Op}(a), t$ fixed. The principal symbol of $A(t)$ is $a \circ F_{t}$ by 1.14. Pick $\left(x_{0}, \xi_{0}\right), \xi_{0} \neq 0$ in local coordinates, and choose any sequence $\rho_{1} \leq \rho_{2} \leq \cdots \rightarrow \infty$ with $\rho_{1}$ already so large that Lemma 1.15 is applicable.

Define $\left(x_{k}, \xi_{k}\right)=\left(x_{t}\left(x_{0}, \rho_{k} \xi_{0}\right), \xi_{t}\left(x_{0}, \rho_{k} \xi_{0}\right)\right), r_{k}=\left|\xi_{k}\right|, \theta_{k}=\xi_{k} /\left|\xi_{k}\right|$. By Lemma 1.15, $x_{k} \rightarrow x_{0}, r_{k} \rightarrow \infty, \theta_{k} \rightarrow \xi_{0} /\left|\xi_{0}\right|$. It follows that

$$
\begin{aligned}
\mid a \circ F_{t}\left(x_{0}, \rho_{k} \xi_{0}\right) & -\sigma(a)\left(x_{0}, \xi_{0} /\left|\xi_{0}\right|\right) \mid \\
= & \left|a\left(x_{k}, \xi_{k}\right)-\sigma(a)\left(x_{0}, \xi_{0} /\left|\xi_{0}\right|\right)\right| \\
= & \left|a\left(x_{k}, r_{k} \theta_{k}\right)-\sigma(a)\left(x_{0}, \xi_{0} /\left|\xi_{0}\right|\right)\right| \rightarrow 0
\end{aligned}
$$

by Lemma $1.5(\mathrm{c})$. Since we know that $\sigma(a)\left(x_{0}, \xi_{0} /\left|\xi_{0}\right|\right)=\sigma(a)\left(x_{0}, \xi_{0}\right)$, we get the desired result.

(b) First recall that $\partial_{t} S(t) f=i M S(t) f$ for arbitrary $f \in C^{\infty}(X)$. Thus $\partial_{t} A(t) f(x)=i[A(t), M] f(x)=i[A, M](t) f(x)$ and

$$
\begin{aligned}
(A(t+h)-A(t)) f(x) & =h \int_{0}^{1} \partial_{t} A(t+u h) f(x) d u \\
& =i h \int_{0}^{1}[A, M](t+u h) f(x) d u .
\end{aligned}
$$


$S(t)$ is bounded and $[A, M]$ is in $\mathscr{P}^{0}$, so the $L^{2}$-norm of the right hand side can be estimated by $h \cdot$ const. $\cdot\|[A, M]\|_{0,0} \cdot\|f\|_{0}$. This shows that $t \mapsto A(t)$ is continuous for $A \in \mathscr{P}^{0}$. In the same way, $[A, M](t)$ is in $\mathscr{P}^{0}$ and continuous in $t$, and so is $[[A, M], M](t)$. Now a similar argument as before proves differentiability: $\partial_{t}^{2} A(t) f(x)=-[[A, M], M](t) f(x)$. Hence

$$
\begin{aligned}
((A(t+h) & -A(t)) / h-i[A, M](t)) f(x) \\
& =h \int_{0}^{1} \int_{0}^{1} \partial_{t}^{2} A(t+u v h) f(x) d u d v \\
& =-h \int_{0}^{1} \int_{0}^{1}[[A, M], M](t+u v h) f(x) d u d v .
\end{aligned}
$$

By estimating the $L^{2}$-norm and letting $h \rightarrow 0$ we get $\partial_{t} A(t)=i[A, M](t)$. This also leads to the desired statement on the symbol.

\subsection{Corollary. Let $A \in \mathscr{P}_{0}$.}

(a) $A(t) \in \mathscr{P}_{0}, \sigma(A(t))=\sigma(A)$. Note that $A(t)$ is well-defined as an operator in $L\left(H^{0}\right)$.

(b) $t \mapsto A(t)$ is continuous.

Proof. (a) $\|A(t)-B(t)\|=\|(A-B)(t)\| \leq$ const. $\|A-B\| . \mathscr{P}_{0}$ is the closure of $\mathscr{P}^{0}$. Thus (a) follows from 2.2(a).

(b) Take a sequence $A_{k}$ in $\mathscr{P}^{0}$ converging to $A$. Since $t \mapsto A_{k}(t)$ is continuous (even differentiable), and the convergence is uniform by the estimate used in the proof of (a), $t \mapsto A(t)$ is also continuous.

2.4. Definition. Let $s$ be an arbitrary function in $\mathscr{S}(\mathbf{R})$, the Schwartz space of rapidly decreasing functions, with $\int s(t) d t=1$, e.g. $s(t)=$ $\pi^{-1 / 2} \exp \left(-t^{2}\right)$, the actual choice of $s$ does not matter. Now define the operator $B$ on $\mathscr{P}_{0}$ by

$$
B(A)=\int A(t) s(t) d t
$$

where all the integrals are taken over $\mathbf{R}$.

2.5. Lemma. (a) The integral in (2.1) exists as an improper Riemann integral, hence $B(A) \in \mathscr{P}_{0}$ for $A \in \mathscr{P}_{0}$.

(b) The map $A \mapsto B(A)$ is continuous on $\mathscr{P}_{0}$.

(c) $\sigma(B(A))=\sigma(A)$.

(d) The commutator $[M, B(A)]$, first considered as an operator in $L\left(H^{1 / 2}, H^{-1 / 2}\right)$ extends to an operator in $L\left(H^{0}\right)$. In fact, $[M, B(A)]=$ $-i \int A(t) s^{\prime}(t) d t$. 
Proof. (a) is a consequence of the boundedness of $S(t)$.

(b) $\|B(A)\| \leq$ const. $\cdot\|A\| s(t) d t \leq$ const. $\cdot\|A\|$.

(c) follows from the continuity of the symbol map:

$$
\sigma(B(A))-\sigma(A)=\int \sigma(A(t)-A) s(t) d t=0,
$$

where we have used that $\int s(t) d t=1$.

(d) By continuity of the integral and Lemma 2.2(b), we have for $A \in \mathscr{P}^{0}$

$$
\begin{aligned}
{[M, B(A)] } & =\int[M, A(t)] s(t) d t=i \int \partial_{t} A(t) s(t) d t \\
& =-i \int A(t) s^{\prime}(t) d t
\end{aligned}
$$

using integration by parts. Letting $A \in \mathscr{P}_{0}, A_{k} \in \mathscr{P}^{0}, A_{k} \rightarrow A$, we get from (b) that $B\left(A_{k}\right) \rightarrow B(A)$ in $L\left(H^{0}\right)$. Thus, on the left hand side, $\left[M, B\left(A_{k}\right)\right] \rightarrow[M, B(A)]$ in $L\left(H^{1 / 2}, H^{-1 / 2}\right)$. On the right hand side, $-i \int A_{k}(t) s^{\prime}(t) d t$ tends to $-i \int A(t) s^{\prime}(t) d t$ (the integrals exist and define operators in $L\left(H^{0}\right)$, because $s^{\prime}$ is in $\left.\mathscr{S}(\mathbf{R})\right)$. This shows that $[M, B(A)]=$ $-i \int A(t) s^{\prime}(t) d t$ is in $L\left(H^{0}\right)$.

2.6. Corollary. Let $j \in \mathbf{N}, A \in \mathscr{P}_{0}$.

(a) $\operatorname{ad}^{j} M(B(A))$ extends to an operator in $L\left(H^{0}\right)$. We have $\operatorname{ad}^{\prime} M(B(A))=(-i)^{j} \int A(t) s^{(j)}(t) d t$.

(b) $\sigma\left(\operatorname{ad}^{j} M(B(A))\right)=0$, i.e. $\operatorname{ad}^{j} M(B(A))$ is compact.

Proof. (a) follows by induction from Lemma 2.5(d).

(b) Using (a) and Lemma 2.3(a), we have $\sigma\left(\operatorname{ad}^{j} M(B(A))\right)=$ $(-i)^{j} \int \sigma(A) s^{(j)}(t) d t=0$, after integration by parts.

2.7. Proof of Theorem 2.1. Let $a \in C\left(S^{*} X\right)$. Pick any operator $P \in \mathscr{P}_{0}$ with $\sigma(P)=a$. Define $A=B(P)$. Then $\sigma(A)=a$ by $2.5(\mathrm{c})$, and $\operatorname{ad}^{j} M(A)$ is compact for each $j \in \mathbf{N}$ by $2.6(\mathrm{~b})$. The operators $M^{-j}$, $j \in \mathbf{N}$, are also compact. Hence $M^{-j} \operatorname{ad}^{j} M(A)$ and $\operatorname{ad}^{j} M(A) M^{-j}$ are compact operators on $H^{0}$ for each $j \in \mathbf{N}$. By 1.7(d), $A$ is in $\mathscr{P}_{\infty}$.

\section{REFERENCES}

[1] M. Atiyah, and I. Singer, The index of elliptic operators I, Ann. Math., 87 (1968), 484-530.

[2] H. O. Cordes, Elliptic Pseudodifferential Operators-An Abstract Theory, Springer Lecture Notes, v. 756, New York, 1979.

[3] __ On compactness of commutators..., J. Funct. Anal., 18 (1975), 115-131. 
[4] H. O. Cordes, and E. Herman, Gelfand theory of pseudodifferential operators, Amer. J. Math., 90 (1968), 681-717.

[5] H. O. Cordes and E. Schrohe, On the symbol homomorphism of a certain Fréchet algebra of singular integral operators, Integral Equations Operator Theory, 8 (1985), 641-649.

[6] H. O. Cordes and D. A. Williams, An algebra of pseudo-differential operators with non-smooth symbol, Pacific J. Math., 78 (1978), 279-290.

[7] B. Gramsch, Relative Inversion in der Störungstheorie von Operator-und $\Psi$-Algebren, Math. Ann., 269 \# 1 (1984), 27-71.

[8] R. T. Seeley, Integro-differential operators on vector-bundles, Trans. Amer. Math. Soc., 117 (1965), 167-204.

[9] _ Complex powers of an elliptic operator, Amer. Math. Soc. Proc. Symp. Pure Math., X (1967), 288-307.

[10] M. E. Taylor, Pseudodifferential Operators, Princeton, 1981.

Received December 4, 1985.

JOHANNES-GUTENBERG-UNIVERSITÄT

SAARSTR. 21

6500 MAINZ, WEST GERMANY 


\section{PACIFIC JOURNAL OF MATHEMATICS EDITORS}

\author{
V. S. VARADARAJAN \\ (Managing Editor) \\ University of California \\ Los Angeles, CA 90024 \\ HERBERT ClEMENS \\ University of Utah \\ Salt Lake City, UT 84112 \\ R. FINN \\ Stanford University \\ Stanford, CA 94305
}

\author{
HERMANN FLASCHKA \\ University of Arizona \\ Tucson, AZ 85721 \\ RAMESH A. GANGOLLI \\ University of Washington \\ Seattle, WA 98195 \\ VAUghan F. R. JONES \\ University of California \\ Berkeley, CA 94720 \\ ROBION KIRBY \\ University of California \\ Berkeley, CA 94720
}

C. C. MOORE

University of California Berkeley, CA 94720

H. SAMELSON

Stanford University Stanford, CA 94305

HAROLD STARK

University of California, San Diego La Jolla, CA 92093

\section{ASSOCIATE EDITORS}
R. ARENS
E. F. BECKENBACH
B. H. NEUMANN
F. WOLF
K. YOSHIDA (1906-1982)

\section{SUPPORTING INSTITUTIONS}

UNIVERSITY OF ARIZONA

UNIVERSITY OF BRITISH COLUMBIA

CALIFORNIA INSTITUTE OF TECHNOLOGY

UNIVERSITY OF CALIFORNIA

MONTANA STATE UNIVERSITY

UNIVERSITY OF NEVADA, RENO

NEW MEXICO STATE UNIVERSITY

OREGON STATE UNIVERSITY
UNIVERSITY OF OREGON UNIVERSITY OF SOUTHERN CALIFORNIA

STANFORD UNIVERSITY

UNIVERSITY OF HAWAII

UNIVERSITY OF TOKYO

UNIVERSITY OF UTAH

WASHINGTON STATE UNIVERSITY

UNIVERSITY OF WASHINGTON 


\section{Pacific Journal of Mathematics}

Vol. 125, No. 1 September, 1986

Gilles Christol, Fonctions et éléments algébriques $\ldots \ldots \ldots \ldots \ldots \ldots \ldots \ldots$

Jo-Ann Deborah Cohen, Extensions of valuation and absolute valued

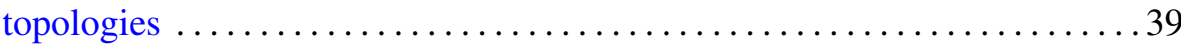

Miriam Cohen, Smash products, inner actions and quotient rings . . . . . . 45

Mikio Furushima, On the singular $K-3$ surfaces with hypersurface

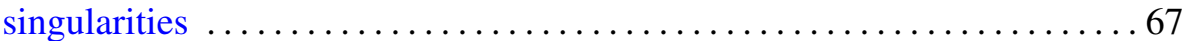

Gerhard Gierz and Boris Shekhtman, A duality principle for rational

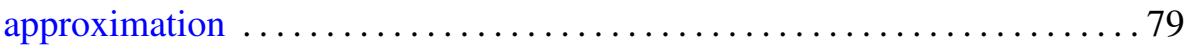

Anthony Wood Hager, A description of HSP-like classes, and

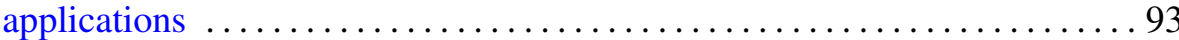

George Alan Jennings, Lines having high contact with a projective

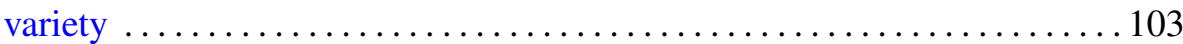

John Lott, Eigenvalue bounds for the Dirac operator . . . . . . . . . . . 117

Denis Laurent Luminet, A functional calculus for Banach PI-algebras . . . . 127

Shizuo Miyajima and Noboru Okazawa, Generators of positive

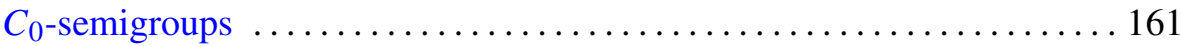

Takemi Mizokami, On functions and stratifiable $\mu$-spaces $\ldots \ldots \ldots \ldots \ldots 177$

Jeff Parker, 4-dimensional $G$-manifolds with 3-dimensional orbits . . . . . 187

Elias Saab and Paulette Saab, On Peł czyński's properties (V) and (V*) . . 205

Elmar Schrohe, The symbols of an algebra of pseudodifferential operators

Aart van Harten and Els Vader-Burger, Approximate Green functions as a tool to prove correctness of a formal approximation in a model of competing and diffusing species

Stephen Watson, Using prediction principles to construct ordered

continua 